\title{
Abstract \\ Participant Engagement with a Hyper-Personalized Activity Tracking Smartphone App
}

Amanda Centi ${ }^{1}$, PhD; Ramya Palacholla ${ }^{1,2,3}$, MD, MPH; Sara Golas ${ }^{1}$, MA; Odeta Dyrmishi ${ }^{1}$, MPA; Stephen Agboola ${ }^{1,2,3}$, MD, MPH; Kamal Jethwani ${ }^{1,2,3}$, MD, MPH; Joseph $\operatorname{Kvedar}^{1,2,3}$, MD

${ }^{1}$ Partners HealthCare, Connected Health Innovation, Boston, MA, United States

${ }^{2}$ Harvard Medical School, Boston, MA, United States

${ }^{3}$ Massachusetts General Hospital, Boston, MA, United States

Corresponding Author:

Amanda Centi, PhD

Partners HealthCare

Connected Health Innovation

25 New Chardon Street, Suite 300, 3rd Floor

Boston, MA,

United States

Phone: 8630091

Email: acenti@partners.org

\section{Abstract}

Background: Many mobile apps have been designed to monitor physical activity. While they may have many downloads, most users eventually stop using the app and become disengaged. We created a hyper-personalized physical activity tracking app to promote engagement with physical activity (PA) among users. It is unknown if this increased engagement and how engagement level may affect measured outcomes.

Objective: The purpose of this study was to determine how users engaged with a hyper personalized activity tracking app for 6 months and whether this engagement affected physical activity

Methods: Participants with cardiometabolic risk (CMR) factors were given an activity watch to track their PA (step counts) and asked to use the study mobile app for 6 months. App features included step tracking, personalized educational and motivational messages, biometric tracking and connection to a portal where their clinician could monitor their activity. App usage data were collected at 3- and 6-month study visits to determine app usage metrics for the 6 months. App engagement was determined by app usage metrics such as overall page clicks (number of clicks per page), frequency of use of individual features (number of clicks) and session length (time spent on a page). Participants were grouped by level of engagement with app (high, medium, low, none) post hoc to determine engagement effects on steps.

Results: Information was collected on 128 participants. Over the 6-month study, 60 participants (47\%) engaged with the app. Among users, app usage decreased by over 50\% with the highest app usage during month 1 followed by month 4 . There was no difference in the average app session lengths at 0 and 6 months (12 vs 11 seconds, respectively). The most commonly viewed feature was the personalized daily messages (92\% of participants used feature, 20,902 clicks, 58\% of total views). At least $85 \%$ of app users engaged with all the features. Each additional day of app use was associated with a nonsignificant increase of 13 steps in overall average daily step count. Median days of app use were used to define groups with high, medium, and low engagement (median 89, 35 and 3 days of use respectively). The low engagement group had an average 1220 less steps per day than the high engagement group $(P<.001)$. High engagement group's session length remained steady through the study period, compared to medium engagement group's session length that fluctuated widely. While steps decreased over the 6 months, those in the medium engagement group decreased in weekly step counts at a steeper slope then other engagement groups.

Conclusions: While participants engaged with most app features, we observed a 50\% decrease in engagement over the 6-month study. Despite this result, those with high engagement were able to achieve more physical activity than those with low engagement. This increase in physical activity may lead to improvements in CMR factors and better quality of life.

(iproc 2018;4(2):e11876) doi: $\underline{10.2196 / 11876}$ 


\section{KEYWORDS}

engagement; physical activity; smartphone

Edited by T Hale; this is a non-peer-reviewed article. Submitted 08.08.18; accepted 29.08.18; published 17.09.18.

Please cite as:

Centi A, Palacholla R, Golas S, Dyrmishi O, Agboola S, Jethwani K, Kvedar J

Participant Engagement with a Hyper-Personalized Activity Tracking Smartphone App

iproc 2018;4(2):e11876

URL: http://www.iproc.org/2018/2/e11876/

doi: $10.2196 / 11876$

PMID:

(C)Amanda Centi, Ramya Palacholla, Sara Golas, Odeta Dyrmishi, Stephen Agboola, Kamal Jethwani, Joseph Kvedar. Originally published in Iproceedings (http://www.iproc.org), 17.09.2018. This is an open-access article distributed under the terms of the Creative Commons Attribution License (https://creativecommons.org/licenses/by/4.0/), which permits unrestricted use, distribution, and reproduction in any medium, provided the original work, first published in Iproceedings, is properly cited. The complete bibliographic information, a link to the original publication on http://www.iproc.org/, as well as this copyright and license information must be included. 